\title{
Amamentação de crianças com necessidades especiais em saúde: Uma revisão integrativa
}

\author{
Breastfeeding of children with special health needs: An integrative review \\ Lactancia materna de niños con necessidades especiales de salud: Una revisión integradora
}

Recebido: 24/06/2021 | Revisado: 04/07/2021 | Aceito: 06/07/2021 | Publicado: 16/07/2021

\author{
Bárbara Hirschmann \\ ORCID: https://orcid.org/0000-0002-6439-3574 \\ Universidade Federal de Pelotas, Brasil \\ E-mail: babi.h@live.com \\ Roberta Hirschmann \\ ORCID: https://orcid.org/0000-0002-3775-0310 \\ Universidade Federal de Pelotas, Brasil \\ E-mail: r.nutri@hotmail.com \\ Ruth Irmgard Bärtschi Gabatz \\ ORCID: https://orcid.org/0000-0001-6075-8516 \\ Universidade Federal de Pelotas, Brasil \\ E-mail: r.gabatz@yahoo.com.br \\ Viviane Marten Milbrath \\ ORCID: https://orcid.org/0000-0001-5523-3803 \\ Universidade Federal de Pelotas, Brasil \\ E-mail: vivianemarten@ hotmail.com
}

\begin{abstract}
Resumo
Esta revisão tem como objetivo conhecer a produção científica sobre as dificuldades e facilidades da amamentação de crianças com necessidades especiais em saúde. Trata-se de uma revisão integrativa realizada nas bases de dados PubMed, Lilacs, BDENF e INDEX Psicologia por meio da combinação dos seguintes descritores: breast feeding OR breastfeeding OR infant AND disabled children OR cerebral palsy OR down syndrome sem restrição de idiomas e período. A pesquisa foi norteada pela seguinte questão: "O que vem sendo produzido sobre as dificuldades e facilidades encontradas no processo de amamentação de crianças com necessidades especiais em saúde?”. Foram selecionados 11 artigos, quatro utilizaram abordagem qualitativa, cinco eram quantitativos e dois eram quantiqualitativos. Dentre os fatores que podem influenciar na amamentação de crianças com necessidades especiais em saúde destacaram-se dificuldades como falta de apoio, desencorajamento, hospitalização e sentimentos negativos e entre as facilidades, experiências prévias com amamentação e apoio dos profissionais de saúde foram relatadas. Ressalta-se a importância do papel da família e dos serviços de saúde na assistência para o estabelecimento do aleitamento materno e sua manutenção.
\end{abstract}

Palavras-chave: Aleitamento materno; Lactente; Crianças com deficiência; Enfermagem; Saúde da criança.

\begin{abstract}
This review aim to know the scientific production about the difficulties and facilities of breastfeeding children with special health needs. This is an integrative review conducted in the PubMed, Lilacs, BDENF and INDEX Psychology databases by combining the following descriptors: breast feeding OR breastfeeding OR infant AND disabled children OR cerebral palsy OR down syndrome without language restriction and period. The research was guided by the following question: "What has been produced about the difficulties and facilities found in the breastfeeding process of children with special health needs?". Eleven articles were selected, four use a qualitative approach, five were quantitative and two were quanti-qualitative. Among the factors that can influence the breastfeeding of children with special health needs, difficulties were highlighted, such as lack of support, discouragement, hospitalization and negative feelings and among the facilities, previous experiences with breastfeeding and support from health professionals were reported. The importance of the role of the family and health services in assisting in the establishment of breastfeeding and its maintenance is emphasized.
\end{abstract}

Keywords: Breast feeding; Infant; Disabled children; Nursing; Child health.

\section{Resumen}

Esta revisión tiene como objetivo conocer la producción científica sobre las dificultades y facilidades de la lactancia materna en niños con necesidades especiales de salud. Se trata de una revisión integradora realizada en las bases de datos PubMed, Lilacs, BDENF e INDEX Psicología mediante la combinación de los siguientes descriptores: lactancia materna $\mathrm{O}$ lactancia materna $\mathrm{O}$ lactante $\mathrm{Y}$ niños discapacitados $\mathrm{O}$ parálisis cerebral $\mathrm{O}$ síndrome de down sin 
restricción de idioma y curso temporal. La investigación estuvo guiada por la siguiente pregunta: “¿Qué se ha producido sobre las dificultades y facilidades encontradas en el proceso de lactancia de niños con necesidades especiales de salud?". Se seleccionaron once artículos, cuatro utilizaron un enfoque cualitativo, cinco fueron cuantitativos y dos fueron cuantitativos-cualitativos. Entre los factores que pueden influir en la lactancia materna de niños con necesidades especiales de salud, se destacaron dificultades como falta de apoyo, desánimo, hospitalización y sentimientos negativos, y entre las facilidades se reportaron experiencias previas con la lactancia materna y el apoyo de los profesionales de la salud. Destacamos la importancia del papel de la familia y los servicios de salud para ayudar al establecimiento de la lactancia materna y su mantenimiento.

Palabras clave: Lactancia materna; Lactante; Niños con discapacidad; Enfermería; Salud del niño.

\section{Introdução}

O leite materno é considerado o único alimento completo e suficiente para o bebê, uma vez que isoladamente é capaz de atender todas as suas necessidades nutricionais durante os primeiros seis meses de vida, proporcionando também maior interação entre ele e sua mãe e o bebê, além de auxiliar no seu desenvolvimento cognitivo e emocional (Brasil, 2019; Frota, Gavião \& Aguiar, 2015).

O aleitamento materno (AM) traz diversos benefícios para o lactente como melhor desenvolvimento da cavidade oral pela sucção, melhor nutrição e aumento da inteligência, ao longo da vida, além de reduzir a incidência de diarreia, alergias, hipertensão, hipercolesterolemia e diabetes (Victora et al., 2015; 2016). Para as lactantes, a amamentação pode proteger contra diabetes tipo 2, câncer de mama e de ovário, além de atuar como método contraceptivo, desde que esteja amamentando exclusiva ou predominantemente e ainda não tenha menstruado (Victora et al., 2016; Brasil, 2019).

O Ministério da Saúde (MS) e a Organização Mundial de Saúde (OMS) recomendam que durante os primeiros seis meses de vida o aleitamento materno seja exclusivo e complementado até no mínimo dois anos de idade, juntamente com alimentos apropriados a essa faixa etária, evitando os prejuízos à saúde mencionados anteriormente (Brasil, 2019; OMS, 2021).

No entanto, o nascimento de um bebê com alguma necessidade especial em saúde, como paralisia cerebral ou Síndrome de Down, pode interferir na lactação pelas características apresentadas por estas crianças como por exemplo, reflexo de deglutição-sucção anormal, hipotonia muscular ou malformações de mandíbula, nariz ou boca (Wieczorkievicz \& Souza, 2009; Brasil, 2015).

Além da situação de saúde do bebê, outros fatores podem influenciar na amamentação como a história familiar, o estado emocional da mãe, assim como o apoio da família, da comunidade, do trabalho, dos profissionais e dos serviços de saúde (Frota et al., 2015).

Nesse processo, o enfermeiro é um dos profissionais de saúde de fundamental importância, uma vez que devido às condições de saúde em que se encontram as Crianças com Necessidades Especiais em Saúde (CRIANES), deve haver orientação adequada e estímulo à mãe quanto aos benefícios do aleitamento materno, bem como maior apoio e assistência de forma a facilitar a amamentação (Batista, Farias \& Melo, 2013). Isso porque na prática, amamentar se torna ainda mais complexo para mães de CRIANES, pois além do cuidado geral de uma criança, as CRIANES requerem e necessitam de cuidados especiais. Sendo assim, o acompanhamento da dupla por uma equipe multiprofissional, favorece o estabelecimento $\mathrm{e}$ a manutenção do aleitamento materno e os cuidados prestados pelos profissionais de saúde, a essa mãe, podem ser essenciais para a lactação (Brasil, 2015; Sooben, 2012).

Estudo mostra que CRIANES são amamentadas por menos tempo quando comparadas às outras crianças (Frota et al., 2015). Além disso, mães de CRIANES muitas vezes são desestimuladas ou desencorajadas a amamentar (Pisacane et al., 2003) ou o fazem pelas dificuldades e receios encontrados nesse processo (Reilly \& Skuse, 1992).

Dessa forma, entendendo a importância da amamentação para a saúde do bebê, bem como para a manutenção do vínculo mãe-filho, busca-se, através de uma revisão integrativa, melhor compreender as facilidades e dificuldades da amamentação de CRIANES, tanto no aspecto do cuidado quanto da vivência das mães nessa etapa, bem como os fatores 
emocionais experienciados por estas. Para tanto, utilizou-se como questão de pesquisa: O que vem sendo publicado sobre as facilidades e dificuldades encontradas no processo de amamentação de CRIANES?

\section{Metodologia}

Trata-se de uma revisão integrativa, delineamento que objetiva reunir resultados encontrados em pesquisas sobre determinado tema ou questão, por meio de um processo sistemático e rigoroso, o qual concede vastas informações sobre um assunto, formando um corpo de conhecimento (Mendes, Silveira \& Galvão, 2019).

Esta revisão integrativa foi conduzida em seis etapas: reconhecimento do tema e seleção da questão de pesquisa; escolha de critérios de inclusão e exclusão de estudos/amostragem ou revisão na literatura; determinação das informações a serem retiradas dos estudos selecionados/categorização dos estudos; análise dos estudos incluídos; interpretação dos resultados; e apresentação da revisão/resumo do conhecimento (Mendes et al., 2019).

A busca dos estudos para comporem a revisão se deu nas bases de dados: National Library of Medicine (PubMed), Literatura Latino-Americana e do Caribe em Ciências da Saúde (LILACS), Base de Dados de Enfermagem (BDENF) e INDEX Psicologia.

A busca incluiu artigos publicados até 22 de agosto de 2020 a partir dos seguintes Descritores em Ciência da Saúde (DeCS): "breast feeding" OR breastfeeding OR infant AND "disabled children" OR "cerebral palsy" OR "down syndrome" nas bases de dados anteriormente descritas, sem restrição de idiomas e período.

Como critério de inclusão usou-se artigos que abordassem a amamentação de CRIANES. Foram excluídos artigos de revisão, teses, dissertações, monografias e aqueles que não tinham relação com o tema da pesquisa.

Ao total foram encontrados 17.373 artigos com os termos utilizados, após a exclusão daqueles que estavam duplicados nas bases de dados. Todos os artigos foram importados para o software EndNote ${ }^{\circledR}$, ferramenta que auxilia na construção do banco de dados e seleção de estudos primários para condução da revisão (Mendes et al., 2019). A partir da leitura dos títulos, foram excluídos aqueles que não tinham relação com o tema da pesquisa. Logo após esta etapa, 67 estudos foram selecionados para a leitura dos resumos, sendo excluídos os que não mencionavam amamentação de crianças com alguma necessidade especial em saúde. Entre os selecionados foram separados 12 estudos para leitura na íntegra, sendo excluído um artigo que não apresentava informações sobre as facilidades e dificuldades na amamentação de CRIANES, totalizando 11 estudos, os quais fazem parte desta revisão, pois atendem os critérios de inclusão e exclusão (Figura 1). A figura 1 apresenta o fluxograma da seleção dos estudos. 
Figura 1. Fluxograma da seleção dos estudos, Brasil. 2021.

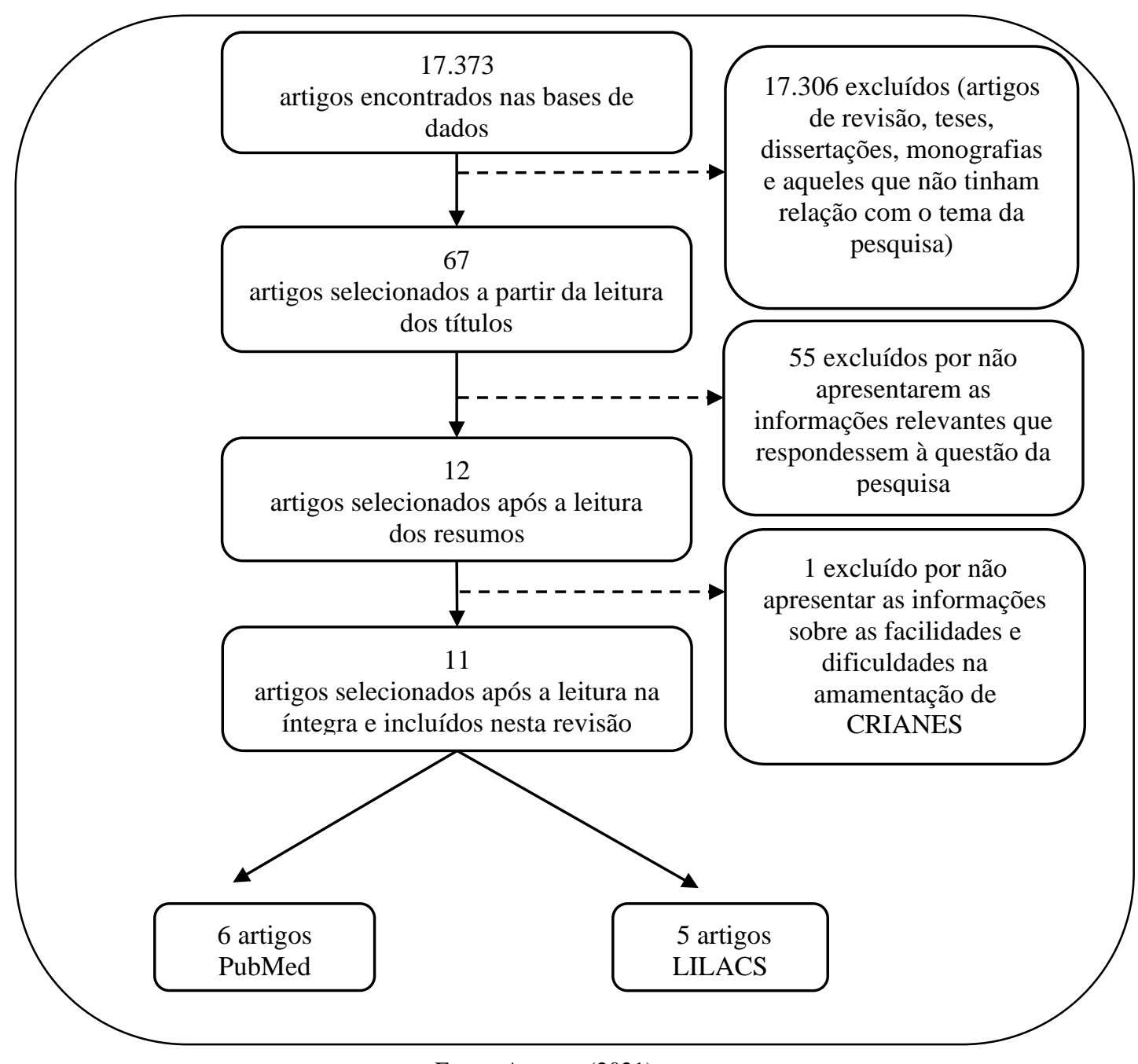

Fonte: Autores (2021).

Os onze artigos que compõem esta revisão foram analisados de acordo com a autoria, local e ano de publicação, objetivos, população, tipo de estudo, resultados/conclusão, base de dados, idioma e nível de evidência avaliado de acordo com Melnyk e Fineout-Overholt (2005), conforme é apresentado no Quadro 1. 
Quadro 1. Classificação do nível de evidência, Brasil. 2021.

\begin{tabular}{|c|l|}
\hline Nível de evidência & \multicolumn{1}{c|}{ Tipo de estudo } \\
\hline I & Revisão sistemática e metassíntese \\
\hline II & Experimentos randomizados ou controlados \\
\hline III & Experimentos controlados sem randomização \\
\hline IV & Estudo de coorte ou caso-controle \\
\hline V & Revisão sistemática de estudos qualitativos ou descritivos \\
\hline VI & Estudos qualitativos ou descritivos \\
\hline VII & Opinião de autoridades ou comitê de especialistas \\
\hline
\end{tabular}

Fonte: Melnyk e Fineout-Overholt (2016).

\section{Resultados e Discussão}

Os artigos incluídos nesta revisão abordaram as facilidades e/ou dificuldades encontradas no processo de amamentação de CRIANES e estão sistematizados no Quadro 2.

O processo de amamentação vai muito além de nutrir a criança, ele também é fundamental para a criação de vínculo com a mãe, favorecendo o desenvolvimento emocional e psíquico do bebê, com a troca de amor e conforto (Nunes, 2015). Independentemente do tipo de aleitamento o vínculo poderá ser construído, pois ele acontece pela interação e pelo contato contínuo entre a dupla (Brasil, 2015).

No entanto, o início da amamentação para muitas mulheres pode ser um processo difícil e, a ausência de apoio (familiar e/ou profissional) nesse período pode levar ao desmame precoce (Brasil, 2015). Isto se torna ainda mais importante quando se pensa em CRIANES as quais demandam um cuidado diferenciado, seja ele temporário ou permanente (Rosseto, Toso, Rodrigues, Vieira, \& Neves, 2019).

Segundo estudo (Barros da Silva, Barbieri-Figueiredo \& Riper, 2018) o processo de lactação de CRIANES é difícil, muitas mulheres não tem a oportunidade de amamentar seus filhos logo após o nascimento, por problemas de saúde ou pelos profissionais acreditarem que a amamentação de CRIANES seria impossível.

No caso de CRIANES, a amamentação pode se tornar ainda mais complexa e difícil, visto que muitas vezes estes bebês podem apresentar reflexo de sucção-deglutição diminuído, protusão da língua, hipotonia muscular, sonolência e/ou malformações orofaciais, necessitando ainda mais de apoio dos familiares e profissionais de saúde para que o AM seja estabelecido. No entanto, para essas crianças o AM é fundamental visto que auxilia, também, no desenvolvimento da musculatura orofacial e na diminuição das ocorrências de infecções (Brasil, 2015). 


\begin{tabular}{|c|c|c|c|c|c|}
\hline $\begin{array}{c}\text { Autor } \\
\text { País, ano }\end{array}$ & $\begin{array}{c}\text { Objetivos/Questão de } \\
\text { pesquisa }\end{array}$ & População & $\begin{array}{l}\text { Tipo de } \\
\text { estudo }\end{array}$ & Resultados/Conclusão & $\begin{array}{l}\text { Base de dados } \\
\text { Idioma } \\
\text { Nível de } \\
\text { Evidência }\end{array}$ \\
\hline $\begin{array}{l}\text { Aumonier, M.E \& } \\
\text { Cunningham, C.C. } \\
\text { Inglaterra, } 1983\end{array}$ & $\begin{array}{l}\text { Quais são as atitudes das } \\
\text { mães em relação à } \\
\text { alimentação antes e após o } \\
\text { nascimento de seus filhos?; } \\
\text { Qual é a extensão das } \\
\text { dificuldades iniciais de } \\
\text { sucção / alimentação em } \\
\text { bebês com Síndrome de } \\
\text { Down?; Até que ponto os } \\
\text { funcionários do hospital } \\
\text { incentivam a amamentação } \\
\text { quando um bebê tem } \\
\text { Síndrome de Down? }\end{array}$ & $\begin{array}{l}59 \text { mães de bebês } \\
\text { com Síndrome de } \\
\text { Down. }\end{array}$ & $\begin{array}{l}\text { Quantitativo } \\
\text { / Qualitativo }\end{array}$ & $\begin{array}{l}31 \text { bebês com Síndrome de Down não tiveram dificuldade em } \\
\text { estabelecer a sucção, quatro foram lentos por menos de } 1 \text { semana, } 8 \\
\text { levaram } 1 \text { semana e } 16 \text { levaram mais de } 1 \text { semana. Anomalia } \\
\text { cardíaca grave foi associada a baixa capacidade de sucção. } \\
\text { Concluiu-se que bebês com Síndrome de Down podem ser } \\
\text { amamentados com sucesso, mas suas mães precisam perseverar e } \\
\text { receber apoio e incentivo durante a internação hospitalar. }\end{array}$ & $\begin{array}{l}\text { PUBMED } \\
\text { Inglês } \\
\text { VI }\end{array}$ \\
\hline $\begin{array}{l}\text { Reilly, S. \& Skuse, D.; } \\
\text { Inglaterra, } 1992\end{array}$ & $\begin{array}{l}\text { Estudar os } \\
\text { alimentares }\end{array}$ de $\begin{array}{r}\text { udrões } \\
\text { uma } \\
\text { amostra que apresentava } \\
\text { alto risco de desordem } \\
\text { alimentar por conta de } \\
\text { disfunção motor-oral e } \\
\text { descrever as práticas } \\
\text { alimentares normais de seu } \\
\text { cuidador habitual em suas } \\
\text { próprias casas. }\end{array}$ & $\begin{array}{l}12 \text { crianças com } \\
\text { paralisia } \\
\text { cerebral/disfunção } \\
\text { motora-oral. }\end{array}$ & $\begin{array}{l}\text { Quantitativo } \\
\text { / Qualitativo }\end{array}$ & $\begin{array}{l}\text { Proporções semelhantes de mães em ambos os grupos começaram a } \\
\text { amamentar seus bebês (oito no grupo casos vs. sete no grupo } \\
\text { comparação). No entanto, seis mães do grupo de casos } \\
\text { interromperam a amamentação por nove semanas por causa de } \\
\text { sucção fraca ou lenta, dificuldade em prender o mamilo ou } \\
\text { empurrá-lo para fora com a língua, e preocupação porque o leite } \\
\text { tendia a escorrer pela boca do bebê. Outros estavam preocupados } \\
\text { com tosse e asfixia excessivas. Houve significativamente mais } \\
\text { dificuldades com sucção precoce e deglutição entre os bebês do } \\
\text { caso. Quando as mães do caso introduziram mamadeiras, problemas } \\
\text { semelhantes foram encontrados. Três mencionaram que as } \\
\text { mamadeiras pareciam piores do que as mamadas, pois seus bebês } \\
\text { "engasgavam mais". Por outro lado, cinco das sete mães do grupo } \\
\text { comparação conseguiram amamentar por mais de nove semanas e } \\
\text { relataram uma transição suave da mama para a mamadeira. }\end{array}$ & $\begin{array}{l}\text { PUBMED } \\
\text { Inglês } \\
\text { VI }\end{array}$ \\
\hline $\begin{array}{l}\text { Amorim, S.T.S.P. et al. } \\
\text { Brasil, } 1999 .\end{array}$ & $\begin{array}{l}\text { Entender a percepção de } \\
\text { mães de crianças com } \\
\text { Síndrome de Down sobre o } \\
\text { aleitamento materno, sua } \\
\text { relação com essa prática e }\end{array}$ & $\begin{array}{lr}14 \quad \text { mães } & \text { de } \\
\text { crianças } & \text { com } \\
\text { Síndrome } & \text { de } \\
\text { Down. } & \end{array}$ & Qualitativo & $\begin{array}{l}\text { Os principais resultados revelaram que, além dos aspectos } \\
\text { relacionados à criança, o impacto causado pela notícia de um filho } \\
\text { deficiente, bem como, a presença ou não de profissionais de saúde } \\
\text { capacitados e sensíveis na orientação e apoio a essas mulheres, } \\
\text { podem ser fatores inibidores ou facilitadores do processo de }\end{array}$ & $\begin{array}{c}\text { LILACS } \\
\text { Português } \\
\text { VI }\end{array}$ \\
\hline
\end{tabular}




\begin{tabular}{|c|c|c|c|c|c|}
\hline & $\begin{array}{l}\text { a atuação do profissional } \\
\text { de saúde nesse contexto. }\end{array}$ & & & $\begin{array}{l}\text { lactação. As mães relatam como dificuldade no processo de } \\
\text { amamentação a falta de apoio dos profissionais e a forma como foi } \\
\text { dada a notícia de que o filho possui uma deficiência e como } \\
\text { facilidade o apoio, orientação e estímulo dos profissionais de saúde, } \\
\text { experiências prévias com aleitamento materno e vontade de } \\
\text { amamentar. }\end{array}$ & \\
\hline $\begin{array}{l}\text { Pisacane, A. et al. } \\
\text { Itália, } 2003\end{array}$ & $\begin{array}{l}\text { Investigar a frequência do } \\
\text { aleitamento materno em } \\
\text { crianças com Síndrome de } \\
\text { Down. }\end{array}$ & $\begin{array}{lrr}560 \text { mães } & \text { de } \\
\text { crianças } & \text { com } \\
\text { Síndrome } & \text { de } \\
\text { Down e outros } \\
\text { dois grupos de } \\
\text { mães de crianças } \\
\text { que } & \text { não } \\
\text { apresentavam } \\
\text { Síndrome } \\
\text { Down. } \\
\end{array}$ & Quantitativo & $\begin{array}{l}\text { A duração da amamentação foi reduzida nas crianças com Síndrome } \\
\text { de Down comparada aos outros grupos. Os principais motivos } \\
\text { relatados pelas mães por não terem amamentado, considerados } \\
\text { como dificuldades, foram a doença dos lactentes, frustração ou } \\
\text { depressão, insuficiência percebida de leite e dificuldade em mamar } \\
\text { para os bebês que não foram admitidos na unidade. }\end{array}$ & $\begin{array}{l}\text { PUBMED } \\
\text { Inglês } \\
\text { IV }\end{array}$ \\
\hline $\begin{array}{l}\text { Al-Sarheed, M. } \\
\text { Arábia Saudita, } 2005\end{array}$ & $\begin{array}{l}\text { Investigar a amamentação } \\
\text { e a introdução de alimentos } \\
\text { sólidos em crianças com } \\
\text { Síndrome de Down. }\end{array}$ & $\begin{array}{lr}225 \quad \text { mães } & \text { de } \\
\text { crianças } & \text { com } \\
\text { Síndrome } & \text { de } \\
\text { Down. } & \end{array}$ & Quantitativo & $\begin{array}{l}\text { Embora } 66,7 \% \text { das mães tenham iniciado a amamentação, } 30 \% \\
\text { delas interromperam a amamentação aos } 3-6 \text { meses de idade da } \\
\text { criança. No geral, mães de crianças com Síndrome de Down } \\
\text { começaram a desmamar entre } 6 \text { e } 9 \text { meses de idade. Observou-se } \\
\text { que mães de crianças com Síndrome de Down amamentavam seus } \\
\text { filhos com frequência e introduziam alimentos sólidos } \\
\text { comparativamente tarde. }\end{array}$ & $\begin{array}{l}\text { PUBMED } \\
\text { Inglês } \\
\text { VI }\end{array}$ \\
\hline $\begin{array}{l}\text { Wieczorkievicz, A.M. \& } \\
\text { Souza, K.V. } \\
\text { Brasil, } 2009\end{array}$ & $\begin{array}{l}\text { Descrever as facilidades } \\
\text { encontradas pelas mães de } \\
\text { crianças com Síndrome de } \\
\text { Down no processo de } \\
\text { amamentação. }\end{array}$ & $\begin{array}{l}6 \text { mães de crianças } \\
\text { com Síndrome de } \\
\text { Down. }\end{array}$ & Qualitativo & $\begin{array}{l}\text { O processo de amamentação apontou para a necessidade de atenção } \\
\text { especial por parte dos serviços e profissionais de saúde, } \\
\text { particularmente da enfermagem. Nesse sentido, a prática de cuidar } \\
\text { dessas mulheres deve compreender intervenções competentes e } \\
\text { sensíveis, do ponto de vista da ação profissional e do fortalecimento } \\
\text { da autonomia da mulher. }\end{array}$ & $\begin{array}{l}\text { LILACS } \\
\text { Português } \\
\text { VI }\end{array}$ \\
\hline $\begin{array}{l}\text { Ryan, K. et al. } \\
\text { Reino Unido, } 2012\end{array}$ & $\begin{array}{l}\text { Explorar os ajustes } \\
\text { emocionais que as } \\
\text { mulheres fazem quando a } \\
\text { doença ou deficiência } \\
\text { crônica do bebê ameaça a } \\
\text { amamentação. }\end{array}$ & $\begin{array}{l}5 \text { mulheres que } \\
\text { amamentam bebês } \\
\text { com condições } \\
\text { crônicas. }\end{array}$ & Qualitativo & $\begin{array}{l}\text { Foram classificados em três temas principais: "Oprimido", } \\
\text { sentimentos de choque e desamparo. O envolvimento nas questões } \\
\text { práticas da amamentação, depender de outras pessoas e receber } \\
\text { apoio emocional delas ajudou; "Sob o reconhecimento", as } \\
\text { mulheres falavam em não serem ouvidas ou levadas a sério. O foco } \\
\text { na condição médica desvalorizou a contribuição da amamentação e } \\
\text { "Lutando pela normalidade", reformular a situação e reajustar metas } \\
\text { e expectativas ajudou a se ajustar à vida "normal". }\end{array}$ & $\begin{array}{l}\text { PUBMED } \\
\text { Inglês } \\
\text { VI }\end{array}$ \\
\hline Frota, F.D.S. et al. & Avaliar $\quad$ o $\quad$ tempo de & 50 crianças com & Quantitativo & A paralisia cerebral foi a deficiência de maior ocorrência no estudo. & LILACS \\
\hline
\end{tabular}




\begin{tabular}{|c|c|c|c|c|c|}
\hline Brasil, 2015 & $\begin{array}{l}\text { aleitamento materno e } \\
\text { identificar possíveis fatores } \\
\text { interferentes em crianças } \\
\text { com deficiência e } \\
\text { fenotipicamente normais. }\end{array}$ & $\begin{array}{l}\text { deficiência e } 50 \\
\text { fenotipicamente } \\
\text { normais. }\end{array}$ & & $\begin{array}{l}\text { As crianças do gênero masculino no grupo dos fenotipicamente } \\
\text { normais receberam aleitamento materno exclusivo por um período } \\
\text { maior de tempo. As mães do grupo dos fenotipicamente normais } \\
\text { apresentavam um grau de escolaridade superior ao das mães do } \\
\text { outro grupo e menos complicações no parto o que podem ter sido } \\
\text { fatores que influenciaram na duração desta prática. }\end{array}$ & $\begin{array}{c}\text { Português } \\
\text { VI }\end{array}$ \\
\hline $\begin{array}{l}\text { Genova, L. et al. } \\
\text { Chile, } 2018\end{array}$ & $\begin{array}{l}\text { Descrever as frequências } \\
\text { de aleitamento materno } \\
\text { exclusivo até } 6 \text { meses de } \\
\text { crianças com Síndrome de } \\
\text { Down no controle do } \\
\text { Programa } \\
\text { monitoramento de saúde de } \\
\text { Pessoas com Síndrome de } \\
\text { Down e identificar os } \\
\text { fatores que influenciam o } \\
\text { abandono do aleitamento } \\
\text { materno exclusivo. }\end{array}$ & $\begin{array}{l}73 \text { binômios } \\
\text { mães/filhos. }\end{array}$ & Quantitativo & $\begin{array}{l}46,6 \%(34 / 73) \text { das mães realizaram o aleitamento materno } \\
\text { exclusivo até } 6 \text { meses ou mais. } 67,1 \%(49 / 73) \text { das crianças } \\
\text { apresentaram alguma patologia ou malformação associada que } \\
\text { influenciou a amamentação. Das } 39 \text { mães que não amamentaram } \\
\text { exclusivamente até } 6 \text { meses, } 25(64,1 \%) \text { foi por motivos da criança. } \\
\text { A hospitalização durante os primeiros } 6 \text { meses de vida foi o fator } \\
\text { mais significativo na cessação do aleitamento materno exclusivo } \\
\text { antes dos } 6 \text { meses (OR }=6,13) \text {. }\end{array}$ & $\begin{array}{l}\text { LILACS } \\
\text { Espanhol } \\
\text { VI }\end{array}$ \\
\hline $\begin{array}{l}\text { Barros da Silva, R.B. et } \\
\text { al. } \\
\text { Portugal, } 2018\end{array}$ & $\begin{array}{lr}\text { Compreender } & \text { as } \\
\text { experiências } & \text { de } \\
\text { amamentação de mães de } & \text { críndrome de } \\
\text { crianças com Síndrome incluindo suas } \\
\text { Down, o } \\
\text { percepções sobre o } \\
\text { processo de amamentação } \\
\text { e suas práticas específicas. }\end{array}$ & $\begin{array}{lr}10 \quad \text { mães } & \text { de } \\
\text { crianças } & \text { com } \\
\text { Síndrome } & \text { de } \\
\text { Down. } & \end{array}$ & Qualitativo & $\begin{array}{l}\text { O sucesso da amamentação depende muito da disposição das mães } \\
\text { e apoio dos profissionais de saúde como enfermeiros. Os resultados } \\
\text { deste estudo sugerem que o apoio de uma equipe multidisciplinar é } \\
\text { essencial para o sucesso da amamentação. É necessária uma maior } \\
\text { conscientização sobre as recompensas e os desafios únicos da } \\
\text { amamentação desses bebês, bem como a maneira como as famílias } \\
\text { lidam com os desafios atuais. }\end{array}$ & $\begin{array}{c}\text { PUBMED } \\
\text { Inglês } \\
\text { VI }\end{array}$ \\
\hline $\begin{array}{l}\text { Aguilar-Cordero, M.J. et } \\
\text { al. } \\
\text { Colômbia, } 2019\end{array}$ & $\begin{array}{l}\text { Avaliar a técnica e a } \\
\text { duração da amamentação } \\
\text { crianças com Síndrome de } \\
\text { Down e grupo comparação, } \\
\text { utilizando o formulário de } \\
\text { observação da } \\
\text { amamentação. }\end{array}$ & $\begin{array}{l}40 \text { recém-nascidos } \\
\text { com Síndrome de } \\
\text { Down e } 40 \text { recém- } \\
\text { nascidos sem } \\
\text { Síndrome de } \\
\text { Down. }\end{array}$ & Quantitativo & $\begin{array}{l}\text { O início da lactogênese foi mais precoce no grupo Síndrome de } \\
\text { Down ( } 92,5 \% \text { nas primeiras } 24 \text { horas } v s .20 \% \text { ). Na duração da } \\
\text { amamentação não foram encontradas diferenças estatisticamente } \\
\text { significativas entre os grupos. Foi observado que as mães de } \\
\text { crianças com Síndrome de Down encontraram mais dificuldades na } \\
\text { técnica de amamentação, no início das mamadas, o que influenciou } \\
\text { na manutenção do aleitamento materno. }\end{array}$ & $\begin{array}{l}\text { LILACS } \\
\text { Inglês } \\
\text { IV }\end{array}$ \\
\hline
\end{tabular}


Sendo assim, além de todos os benefícios do AM, o vínculo da mãe com o filho com alguma necessidade especial se torna fundamental para que o cuidado seja efetivo, pois é neste momento da amamentação que mãe e filho se conectam criando assim uma ligação afetiva ou apego (Brasil, 2015; Oliveira \& Poletto, 2015).

Nos estudos incluídos nesta revisão ficou evidente a presença de sentimentos negativos uma vez que tristeza, culpa, frustração, impotência e até mesmo raiva foram relatados pelas mães, além do choque de receber a notícia de ter um filho com necessidades especiais. Esses sentimentos, em alguns casos, são determinantes e podem acabar dificultando ainda mais a amamentação (Amorim, Moreira \& Carraro, 1999; Barros da Silva et al., 2018). O nascimento de uma criança é permeado de significados e quando se trata de uma CRIANES os pais se deparam com uma situação difícil, que gera um impacto emocional, pois rompe com a ideia do filho idealizado. A perda do filho imaginado e o nascimento do filho real agrega uma gama de sentimentos complexos e por vezes ambivalentes (Milbrath, Motta, Gabatz, \& Freitag, 2017; Vaz et al., 2018). Os pais deparam-se com medo, ansiedade e tristeza por estarem vivendo um momento inesperado e desconhecido, uma vez que essa situação possivelmente demandará tratamentos e cuidados contínuos (Milbrath et al., 2017; Vaz et al., 2018).

Entre as dificuldades relacionadas à amamentação destacadas pelos autores, além dos problemas emocionais, considerados comuns nesse processo, como a insegurança por acreditar não estar preparada (Ryan, Smith \& Alexander, 2015), decepção, ansiedade de separação, desamparo, vulnerabilidade e culpa também foram mencionados pelas mães. Os autores significaram o processo de ajuste desses sentimentos, de certa forma semelhante ao luto, pelo qual as mulheres passaram ao aceitar a doença crônica ou incapacidade e seu impacto no desejo de amamentar seu bebê (Ryan et al., 2015).

Algumas mães relatam ainda que a notícia de que o filho tem alguma necessidade especial e a falta de apoio profissional podem interferir no processo de amamentação (Amorim et al., 1999). Em alguns casos, o estresse de lidar com emoções diferentes leva a uma certa dificuldade na prática.

De acordo com Amorim et al., (1999) diversos fatores, como a ansiedade e a forma como a notícia sobre a doença crônica da criança é passada para a mãe contribuem para a manutenção da amamentação ou para o desmame precoce, visto que o conhecimento sobre esta condição poderá causar um grande impacto emocional na vida da família. Conforme Brunoni et al., (2016), a repercussão do nascimento e o cuidado de uma CRIANES altera a rotina familiar e afeta diretamente a vida de seus cuidadores. Nesse sentido, os profissionais de enfermagem, por meio das experiências vividas podem realizar o cuidado da dupla mãe-bebê, buscando auxiliar no processo de amamentação (Milbrath et al., 2017), o qual tem importância pois promove o vínculo entre ambos, aproximando-os, além de ser um momento prazeroso e de grande interação (Brasil, 2015). Ainda, o saber ouvir e, sobretudo, o compartilhamento da decisão com a mãe sobre a melhor conduta em relação à alimentação de seu filho são aspectos que devem ser considerados pelo profissional de saúde (Amorim et al., 1999).

A necessidade de hospitalização das CRIANES também foi um fator que interferiu no AM visto que muitas vezes a condição especial de CRIANES faz com que elas precisem ficar hospitalizadas por um tempo maior (Genova, Cerda, Correa, Vergara, \& Lizama, 2018).

As CRIANES requerem cuidados contínuos e complexos da família e de profissionais especializados uma vez que, dependendo da complexidade do seu diagnóstico, necessitam de internações frequentes, as quais podem ser prolongadas (Neves et al., 2015). Dessa forma, destaca-se a importância da reorganização dos serviços de saúde e a assistência a essas famílias no cuidado de CRIANES, dado o crescimento dessa população (Astolpho, Okido \& Lima, 2014).

Quanto a duração da amamentação, os estudos apresentam diferentes resultados. Um deles observou que crianças com Síndrome de Down foram amamentadas por 54 dias em comparação a 164 dias em crianças que não apresentavam a síndrome (Pisacane et al., 2003). Em outro estudo (Frota et al., 2015), os pesquisadores encontraram que o número de mães que amamentaram exclusivamente até os seis meses praticamente não diferiu entre o grupo de crianças que apresentavam alguma 
deficiência com características físicas alteradas, daquelas crianças fenotipicamente sem alteração e destacam que as prevalências foram insatisfatórias nos dois grupos, não atingindo a recomendação da OMS.

O estudo de Aguilar-Cordero et al., (2019), avaliou a duração e a técnica de amamentação e encontrou que $60 \%$ das mães do grupo das crianças que não apresentavam síndrome e 47,5\% das mães do grupo com Síndrome de Down mantiveram o AM por mais de três meses, porém, não foi observada diferença estatisticamente significativa entre os grupos em relação ao tempo de amamentação. No entanto é importante destacar que grande parte da amostra amamentou seus bebês por um período inferior a três meses. Isso demonstra, em ambos os estudos, que além das dificuldades inerentes ao processo de amamentação de CRIANES, há outros fatores que podem também interferir na prática.

Com relação as causas para a cessação do AM, os estudos destacaram alguns motivos maternos como doença da mãe, retorno ao trabalho/estudos, medo de insuficiência de leite e problemas com a criança como dificuldade na amamentação (posturais e de sucção-deglutição), indicação médica de fórmula apesar de bom aumento do peso (Pisacane et al., 2003; Genova et al., 2018; Aguilar-Cordero et al., 2019). Além disso, erros técnicos de amamentação, no início das mamadas, podem influenciar negativamente na manutenção do AM (Aguilar-Cordero et al., 2019).

Pesquisa realizada com crianças com paralisia cerebral mostrou que a amamentação precisou ser interrompida quando as crianças tinham nove semanas por causa de sucção fraca, dificuldade do bebê em prender o mamilo ou empurrá-lo para fora com a língua, e preocupação porque o leite tendia a escorrer pela boca do bebê. Além disso, havia preocupação com tosse e asfixia excessivas. No entanto, foi encontrado também que apesar das dificuldades na amamentação, as mamadeiras pareciam piores que as mamadas, pois provocavam mais engasgos no bebê (Reilly \& Skuse, 1992).

Outro estudo que avaliou crianças com Síndrome de Down apontou que, em geral, a amamentação foi interrompida antes dos seis meses de idade, sendo a duração da amamentação influenciada pela idade da mãe. Entre as razões pelas quais as mães foram desencorajadas a amamentar estavam: pensar que a fórmula tem mais calorias e que o bebê ingere melhor com a mamadeira (Al-Sarheed, 2005).

Em geral, a amamentação de CRIANES não é realizada durante o período preconizado pelo MS e OMS, podendo ser devido às características físicas do bebê como também pela falta de conhecimento sobre o assunto que pode causar insegurança no processo.

Por outro lado, no estudo realizado por Aumonier e Cunningham (1983), 29 mães de crianças com Síndrome de Down desejavam amamentar e, dessas, 16 tiveram sucesso. Além disso, nenhuma dessas mães citou a Síndrome de Down como causa da falha, sendo possível inferir que bebês com Síndrome de Down podem ser amamentados com sucesso, independentemente da doença, mas reforçam a importância da persistência das mães e do apoio e incentivo hospitalar (Aumonier \& Cunningham, 1983). No entanto, o estudo não menciona o tempo durante o qual essas crianças foram amamentadas.

Wieczorkievicz e Souza (2009) reforçam que um fator importante para o efetivo aleitamento é o desejo da mãe, pois mesmo sentindo dor e angústia pelo nascimento do filho com necessidades especiais elas continuam tentando amamentar. Nesse processo, torna-se fundamental o apoio familiar e profissional para ajudar a gerenciar as diferentes emoções, propiciando gerar sentimentos positivos e, assim, contribuir para que a amamentação seja mantida (Barros da Silva et al., 2018).

Os enfermeiros têm um papel fundamental no AM, no auxílio às mães na prática e na manutenção da amamentação, podendo influenciar no sucesso ou fracasso do AM, portanto é de suma importância que esses profissionais estejam preparados para atender as mães de CRIANES, a fim de que a amamentação se torne efetiva (Barros da Silva et al., 2018). Dessa forma, a participação da equipe de enfermagem no apoio a essas famílias é imprescindível, uma vez que o nascimento de uma CRIANES demanda um cuidado diferenciado, altera a rotina familiar causando, muitas vezes, sobrecarga aos cuidadores. 
Com relação as facilidades no processo de amamentação de CRIANES os estudos incluídos nesta revisão observaram que o apoio e o estímulo dos profissionais de saúde, principalmente dos enfermeiros, estavam entre os fatores mencionados assim como as experiências prévias com amamentação (Amorim et al., 1999; Wieczorkievicz \& Souza, 2009; Ryan et al., 2015). Vale ressaltar também a persistência e o desejo da mãe em relação a prática e manutenção do AM, bem como a importância desse contato mãe-bebê e a disseminação do conhecimento sobre o assunto para continuidade da amamentação como forma de intensificar o vínculo e o apego e influenciar positivamente na nutrição da criança, resultando em benefícios para a saúde física e emocional.

Alguns pontos observados nos estudos incluídos nesta revisão merecem ser destacados. Nesta pesquisa foram encontrados apenas artigos publicados após o ano de 1983, o que pode estar relacionado a criação das políticas de amamentação, uma vez que somente a partir da década de 1980 foram implantados no Brasil programas e políticas favoráveis ao AM. Em 1983 a Rede International Baby Food Action Network (IBFAN), que objetiva promover a amamentação e disseminar informações sobre a importância do AM, iniciou suas atividades no país contribuindo para a construção da Norma Brasileira de Comercialização de Alimentos de 1992, que buscou promover práticas saudáveis na alimentação de lactentes e obrigava os fabricantes de fórmulas infantis e produtos que dificultam a amamentação a mencionarem em seus rótulos a importância do AM (Brasil, 1992; Santiago, 2013; IBFAN, 2021).

Somado a isto, também no ano de 1983 foi criado pelo MS o Programa de Assistência Integral à Saúde da Mulher e da Criança com o objetivo de aprimorar as condições de saúde e melhorar a prestação do cuidado nos serviços de saúde à essa população. No ano seguinte, foi elaborado um programa específico para a saúde da criança, o Programa de Assistência Integral à Saúde da Criança, buscando reduzir a morbimortalidade infantil e melhorar as condições de saúde através do aumento da cobertura e da capacidade de resolução dos serviços (Brasil, 2011).

Além disso, de maneira geral, poucos estudos foram encontrados na literatura acessada apesar da relevância desta temática. Dessa forma, salienta-se a importância da elaboração de novos estudos que avaliem o processo de amamentação de CRIANES, uma vez que conhecer as vivências, bem como as dificuldades encontradas por estas mães nesse processo, pode auxiliar os enfermeiros no suporte a essas famílias, tanto emocional quanto fisicamente, visto que os cuidados prestados pelos profissionais de saúde podem ter reflexos positivos no estabelecimento e na manutenção da amamentação de CRIANES.

\section{Conclusão}

O estabelecimento do aleitamento materno é um processo complexo para muitas mães e, quando se trata de amamentação de CRIANES, torna-se ainda mais desafiador. A presente revisão mostrou que o apoio dos profissionais de saúde, as experiências prévias e ao desejo da mãe em amamentar foram destacadas como facilidades no processo de amamentação de CRIANES.

Além disso, observou-se que outros fatores podem influenciar dificultando a amamentação na prática, como falta de apoio, desencorajamento, maior tempo de hospitalização, dificuldades em função da condição crônica do bebê e sentimentos negativos que podem acompanhar esse período, como medo, tristeza, ansiedade, vulnerabilidade, cansaço e raiva, bem como erros na técnica de amamentação podem influenciar negativamente nesse processo. Sendo assim, destaca-se a importância da família e dos profissionais de enfermagem, na assistência a dupla mãe-bebê para o estabelecimento do aleitamento materno e sua manutenção em CRIANES.

\section{Referências}

Aguilar-Cordero, M. J., Rodríguez-Blanque, R., Sánchez-López, A., León-Ríos, X. A., Expósito-Ruiz, M., \& Mur-Villar, N. (2019). Assessment of the technique of breastfeeding in babies with Down Syndrome. Aquichan, 19(4), 1-12. 
Al-Sarheed, M. (2005). Feeding Habits of Children with Down's Syndrome Living in Riyadh, Saudi Arabia. J Trop Pediatr, 52(2), 83-6.

Amorim, S. T. S. P., Moreira, H., \& Carraro, T. E. (1999) Amamentação em crianças com síndrome de down: a percepção das mães sobre a atuação dos profissionais de saúde. Rev Nutr, 12(1), 91-101.

Astolpho, M. P., Okido, A. C. C., \& Lima, R. A. G. (2014). Rede de cuidados a criança com necessidades especiais de saúde. Rev Bras Enferm, 67(2), 213-9.

Aumonier, M. E. \& Cunningham, C. C. (1983). Breast feeding in infants with Down's syndrome. Child care health dev, 9, 247-55.

Barros da Silva R., Barbieri-Figueiredo, M. C., \& Riper, M. V. (2018) Breastfeeding experiences of mothers of children with down syndrome. Compr Child Adolesc Nurs, 1-15.

Batista, K. R. A., Farias, M. C. A. D., \& Melo, W. S. N. (2013) Influência da assistência de enfermagem na prática da amamentação no puerpério imediato. Saúde Debate, 37(96), 130-138.

Brasil. Ministério da Saúde. Departamento de Atenção Básica. (2015) Saúde da Criança: Aleitamento materno e alimentação complementar. Caderno de Atenção Básica. 2 ed. n. 23. Brasília: Ministério da Saúde; 184 p.

Brasil. Ministério da Saúde. (1992). Resolução CNS/MS no 31, de 12 de outubro de 1992. Ministério da Saúde.

Brasil. Ministério da Saúde. Secretaria de Atenção à Saúde. Departamento de Ações Programáticas Estratégicas. Área Técnica de Saúde da Criança e Aleitamento Materno. (2011). Gestões e gestores de políticas públicas de atenção à saúde da criança: 70 anos de história. Brasília: Ministério da Saúde; 80p.

Brasil. Ministério da Saúde. Secretária de Atenção Primária à Saúde. Departamento de Promoção da Saúde. (2019). Guia alimentar para crianças brasileiras menores de 2 anos. Brasília: Ministério da Saúde, 265 p.

Brunoni, D., Blascovi-Assis, S. M., Osório A. A. C., Seabra, A. G., Amato, C. A. H., \& Teixeira, M. C. T. V., Rocha, M. M., \& Carreiro, L. R. R. (2016). Microcefalia e outras manifestações relacionadas ao vírus zika: impacto nas crianças, nas famílias e nas equipes de saúde. Ciênc Saúde Colet, 21(10), 3297302.

Frota, F. D. S., Gavião, M. B. D., \& Aguiar, S. M. H. C. A. (2015). Fatores associados à amamentação em crianças com deficiência e fenotipicamente normais. Rev CEFAC, 17(1), 201-8.

Genova, L., Cerda, J., Correa, C., Vergara, N., \& Lizama, M. (2018). Buenos indicadores de salud em niños com síndrome de Down: Alta frecuencia de lactancia materna exclusiva a los 6 meses. Rev Chil Pediatr, 89(1), 32-41.

IBFAN Brasil [homepage na internet]. IBFAN. http://www.ibfan.org.br/site/

Melnyk, B. M., \& Fineout-Overholt, E. (2005). Making the case for evidence-based practice. In: Melnyk BM, Fineout-Overholt, E. Evidence-based practice in nursing \& healthcare: a guide to best practice [Internet]. Philadelphia: Lippincot Williams \& Wilkins, 3-24.

Mendes, K. D. S., Silveira, R. C. C. P., \& Galvão, C. M. (2019). Uso de gerenciador de referências bibliográficas na seleção dos estudos primários em revisão integrativa. Texto \& Contexto Enferm, 28, 1-13.

Milbrath, V. M., Motta, M. G. C., Gabatz, R. I. B., \& Freitag, V. L. (2017). O nascimento de um filho com paralisia cerebral: um tempo presente inesperado. Rev Interd em Cult e Soc, 3(esp), 47-60.

Neves, E. T., Silveira, A., Arrue, A. M., Pieszak, G. M., Zamberlan, K. C., \& Santos, R. P. (2015). Rede de cuidado de crianças com necessidades especiais de saúde. Texto \& Contexto Enferm, 24(2), 399-406.

Nunes, L. M. (2015). Importância do aleitamento materno na atualidade. Bol Cient Pediatr, 4(3), 55-8.

Oliveira, I. G., \& Poletto, M. (2015). Vivências emocionais de mães e pais de filhos com deficiência. Rev SPAGESP, 16(2), $102-19$.

Organização Mundial da Saúde [homepage na internet]. Breastfeeding. http://www.who.int/topics/breastfeeding/en/

Pisacane, A., Toscano, E., Pirri, I., Continisio, P., Andria, G., Zoli, B., Strisciuglio, P., Concolino, D., Piccione, M., Giudice C.Lo., \& Vicari, S. (2003). Down syndrome and breastfeeding. Acta Pediatric, 92, 1479-81.

Reilly, S., \& Skuse, D. (1992). Characteristics and management of feeding problems of young children with cerebral palsy. Dev Med Child Neurol, 34, 37988.

Rossetto, V., Toso, B. R. G. O., Rodrigues, R. M., Vieira, C. S., \& Neves, E. T. (2019). Cuidado desenvolvido às crianças com necessidades especiais de saúde nos serviços de atenção domiciliar no Paraná - Brasil. Esc Anna Nery Rev Enferm, 23(1), 1-10.

Ryan, K., Smith, L., \& Alexander, J. (2012). When baby's chronic illness and disability interfere with breastfeeding: Women's emotional adjustment. Midwifery, 2, 794-800.

Santiago, L.B. (2013). Manual de aleitamento materno. Manole.

Silva, C. M. S., Bortoli, C. F. C., Massafera, G. I, Silverio, M., Bisognin, P., \& Prates, L. A. (2015). Sentimentos e vivências maternas associadas ao processo de amamentação. Rev Enferm UFPE, 9 (supl 8), 9343-51.

Sooben, R. D. (2012). Breastfeeding patterns in infants with Down's syndrome: A literature review. British Journal of Midwifery, 20(3), 187-92.

Vaz, J. C., Milbrath, V. M., Gabatz, R. I. B., Krug, F. R., Hirschmann, B., \& Oliveira, M. M. (2018). Cuidado à família da criança com doença crônica. Rev Enferm UFPE, 12(5), 1397-408. 
Research, Society and Development, v. 10, n. 8, e48410817542, 2021

(CC BY 4.0) | ISSN 2525-3409 | DOI: http://dx.doi.org/10.33448/rsd-v10i8.17542

Victora, C., Barros, A. J. D., França, G. V. A., Bahl, R., Rollins, N. C., Horton, S., Krasevec, J., Murch, S., Sankar, M. J., \& Walker, N. (2016). Amamentação no século 21: epidemiologia, mecanismos, e efeitos ao longo da vida. Epidemiol Serv Saúde, 1-24.

Victora, C. G., Horta, B. L., Mola, C. L., Quevedo, L., Pinheiro, R. T., Gigante, D. P., Gonçalves, H., \& Barros, F. C. (2015). Association between breastfeeding and intelligence, educational attainment, and income at 30 years of age: a prospective birth cohort study from Brazil. Lancet Glob Health, 3 , 199-205.

Wieczorkievicz, A. M., \& Souza, K. V. (2009). O processo de amamentação de mulheres mães de crianças portadoras de síndrome de down. Cogitare Enferm, 14(3), 420-7. 